Ma. Victoria P. Pascual, MD, MBA

Department of Otorhinolaryngology Head and Neck Surgery

The Medical City

\section{Stridor at Birth: Congenital Laryngeal Web}

Laryngeal web is a rare entity, constituting $5 \%$ of all congenital laryngeal lesions, with a reported incidence of 1 in 10,000. It usually presents with stridor in childhood, but can be discovered in asymptomatic adults under anesthesia and is associated with failed intubations. ${ }^{2}$ We present the case of a newborn with stridor and respiratory distress due to laryngeal web.

\section{CASE REPORT}

A 47-day-old boy was referred to our institution for stridor. He was spontaneously born term at 40 3/7 weeks gestational age to a 26-year-old G2P2 (2002). Birth weight was 3104 grams and maturity testing at 39 weeks was appropriate for gestational age. Prenatal and perinatal history was unremarkable.

Upon delivery with an initial Apgar score of 5 becoming 6, inspiratory stridor and impending respiratory failure prompted intubation. Post-intubation chest $x$-ray revealed minimal lung disease and the baby was extubated after 24 hours. Six hours after extubation, stridor was noted again and the baby was reintubated. A chest $x$-ray showed atelectasis and the baby was managed as a case of pulmonary hypertension. He was weaned from ventilatory support and continuous positive airway pressure (CPAP) was commenced on day 15. Lavage feeding was started on day 18 and 7-day empiric antibiotics were completed. However, stridor and respiratory distress persisted, and the baby was reintubated and recommitted to a mechanical ventilator. Laryngomalacia was suspected, a tracheostomy was recommended and the baby was referred to our institution for further work-up and management. Our admitting impression was a term baby boy with pneumonia, and laryngomalacia versus tracheomalacia.

Upon arrival at our institution, chest $\mathrm{x}$-ray showed hazy and reticular opacities at the posterobasal segment of both lower lung lobes, more on the right, and interpreted as bilateral pneumonia. Ampicillin and Cefotaxime were started, and gram stain, culture and sensitivity of endotracheal secretions resulted in moderate growth of $S$. marscescens and light growth of $K$. pneumonia, both resistant to Ampicillin. Antibiotics were shifted to Gentamycin and Ceftazidime, given for 10 days.

During this time, the baby was also exhibiting myoclonic upper extremity movements but was subsequently cleared for seizures or other structural brain pathology by Neurology. Our plan for airway evaluation was initial flexible endoscopy followed by bronchoscopy if extubation was tolerated. The possibility of a tracheostomy was considered if extubation would not be tolerated.

Flexible endoscopy revealed patent nasal airways with no demonstrable nasal obstruction or structural abnormalities. The endotracheal and orogastric tubes were visualized entering the trachea and esophagus respectively. (Figure 1) There was pooling of secretions in the hypopharyngeal area, but no visible masses or lesions.

Philipp J Otolaryngol Head Neck Surg 2015; 30 (2): 59-6

(C) Philippine Society of Otolaryngology - Head and Neck Surgery, Inc 


\section{FEATURED GRAND ROUNDS}

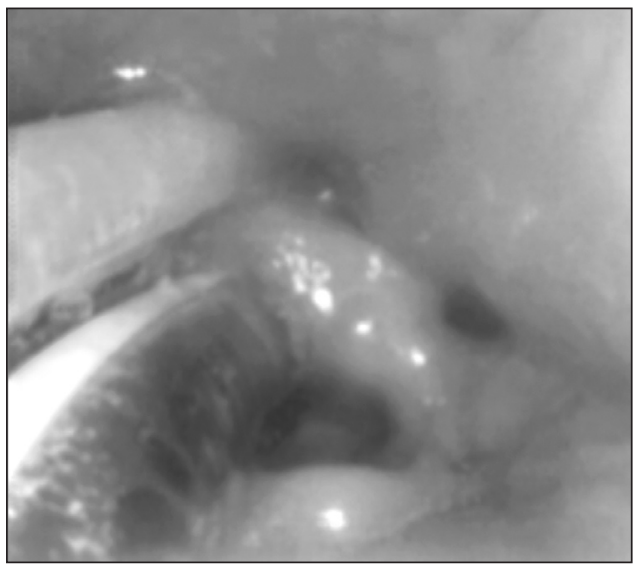

Figure 1. Flexible nasal endoscopy showing the endotracheal tube (clear tube with white stripe) inserted into the airway and the orogastric tube (opaque) inserted into the esophagus. The rest of the surrounding structures were unremarkable.

After extubation, a soft tissue band was visualized traversing the right and left true vocal cords posteriorly, consistent with an interarytenoid web. (Figure 2) A space between this band and the interarytenoid area corresponded to the site where the ET tube had passed. The arytenoid mucosa also appeared swollen and edematous. The epiglottis was
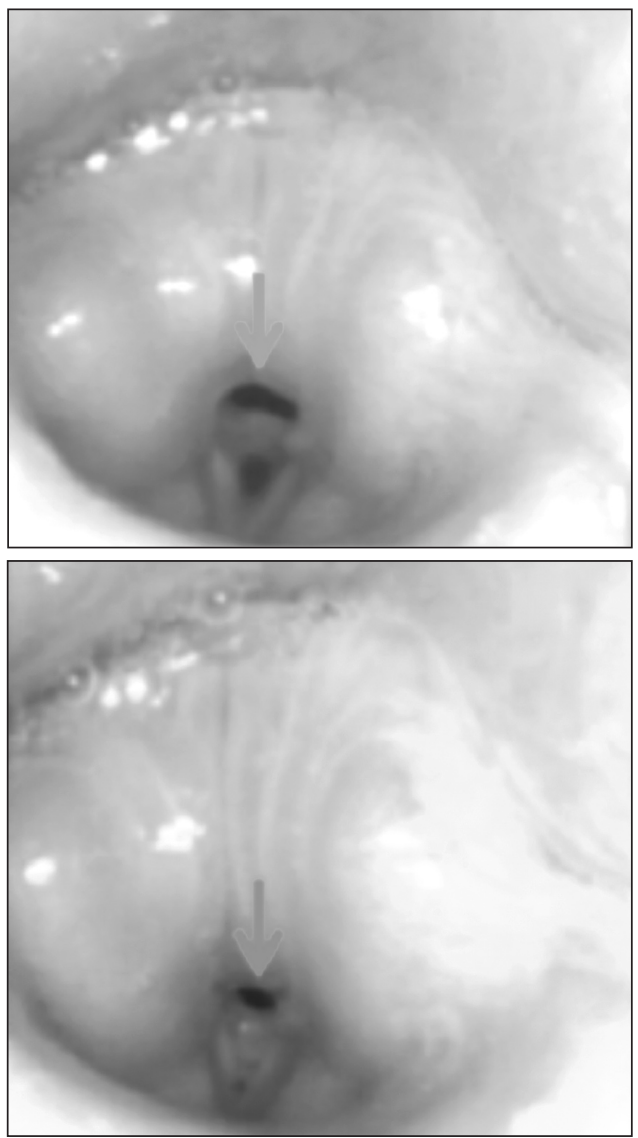

Figure 2. Flexible laryngoscopy after extubation. Top photo shows vocal folds in abduction and bottom photo in adduction. Note the soft tissue band (interarytenoid web) traversing the posterior portion of the vocal folds. Arrow points to space where endotracheal tube previously passed. normal. There was vocal fold motion with incomplete glottic closure, but the full extent of glottic opening and closing could not be assessed due to the band. Because of decreasing oxygenation and episodes of desaturation, the baby was reintubated and a tracheostomy was performed.

The baby was weaned off the ventilator a week later, and transferred out of intensive care to a regular room where he tolerated room air. Clotrimazole that had been given for light growth of S. maltophilia obtained intraoperatively was shifted to Levofloxacin and the last dose was given after 7 days. His postoperative course was unremarkable, and he was discharged with a tracheostomy and nasogastric tube, with suck and swallow therapy for eventual oral feeding. Regular monitoring and routine tracheotomy care with periodic tube changes and endoscopic surveillance of the web and signs of reflux are scheduled.

\section{DISCUSSION}

The most likely conditions for stridor presenting at birth are congenital structural anomalies like laryngomalacia $(60 \%)$, vocal cord paralysis (15-20\%), congenital subglottic stenosis (15\%), laryngeal web $(5 \%)$, or subglottic hematoma $(1.5 \%))^{1,2,3}$

The larynx develops from the endodermal lining and the adjacent mesenchyme of the foregut between the fourth and sixth branchial arches. The arytenoid swelling is formed at the cranial end of the laryngotracheal tube by the proliferation of the mesenchymal tissue (derived from neural crest cells). It grows towards the tongue and forms the primordial glottis. As it grows further, it changes the primordial glottis into a T-shaped laryngeal inlet. ${ }^{4}$

Congenital laryngeal webs are uncommon, constituting $5 \%$ of all congenital laryngeal lesions; their incidence has been estimated at approximately 1 in 10,000 births. ${ }^{4}$ They are due to incomplete recanalization of the laryngotracheal tube during the third month of gestation, leading to different degrees of laryngeal webs. The most common site of development is at the level of the vocal folds anteriorly, although they may occur in the posterior interarytenoid or in the subglottic or supraglottic area. ${ }^{5}$ Diagnosis may be made via flexible or rigid laryngoscopy, or airway films if subglottic or cricoid pathology is present. $^{4}$

Most congenital webs present at birth or in the first few months of life. Symptoms range from mild dysphonia to significant airway obstruction, depending on the size of the web. Hoarseness, croup, and dysphagia are some other symptoms. A third of children with laryngeal webs have anomalies of the respiratory tract, most commonly subglottic stenosis. When congenital in origin, this may be associated with various syndromes like Di-George syndrome, velocardiofacial (Shprintzen) 
syndrome, conotruncal anomaly face syndrome.,

Laryngeal webs may be classified according to airway obstruction. A T1 web is uniform in thickness with no subglottic extension, has true vocal cords clearly visible in the web, usually has no airway obstruction, and hoarseness as the only usual presenting sign. A T2 web is slightly thicker, with a significantly thicker anterior component, and may have minimal subglottic involvement, and a usually husky voice. A T3 web is thick with a solid anterior portion that extends into the subglottis, the true vocal cords are not well delineated, and there is marked vocal dysfunction, with a weak and whispery voice. A T4 web is uniformly thick and extends into the subglottic area with resulting subglottic stenosis. Respiratory obstruction is severe, and the patient is almost always aphonic. ${ }^{4,7}$ Webs may also be classified according to location, whether anterior, posterior (interarytenoid), subglottic, or supraglottc. ${ }^{4}$ Our patient had a type 1, interarytenoid laryngeal web.

About $75-90 \%$ of laryngeal webs are located anteriorly and extend toward the arytenoids. Occasionally, a minor web will not be diagnosed until the child is older and undergoes evaluation for chronic hoarseness. ${ }^{1}$ It may vary in thickness, and the boundary is the vocal process. ${ }^{5}$ Posterior webs may present with apparent bilateral vocal cord paralysis, especially if an interarytenoid web in the posterior larynx limits vocal fold abduction. This type of congenital web is rare and often necessitates a tracheotomy in the early years of life. Stridor is the major presenting clinical feature (as in our case), but patients can also present with obstructive cyanosis at birth or episodes of apnea. ${ }^{2}$

Asymptomatic patients do not require treatment. Treatment depends on the severity of airway obstruction, and may be single or multi-staged. If a patient presents with difficulty breathing, the airway must first be secured. This can be done through endotracheal intubation, which can be converted into a tracheostomy if prolonged intubation is expected. ${ }^{6}$ Long-term tracheotomy with observation for eventual decannulation after 3 to 5 years may be practiced. Surgical division can be achieved using laryngeal knives, microscissors, galvanocautery or radiofrequency. However, these are frequently unsuccessful as vocal cords re-adhere in the area where the web was separated. ${ }^{4,8}$ Surgical correction results in two opposing surfaces with denuded epithelium that tend to heal together and reform a web. To prevent re-adhesion, a keel, stent or probe may be positioned between the two raw edges. Steroid injections or mitomycin may also diminish re-adhesion. ${ }^{2}$ Extensive webs often require arytenoidectomy or even open laryngeal reconstruction to correct subglottic stenosis. This can be done with a costal graft, a posterior cricoid split, or a T-tube. ${ }^{9}$

Given that our patient has a type 1 laryngeal web with a thin band, some may suggest that we observe the patient for 3-5 years in the hope that it might be outgrown. ${ }^{3}$ Others would divide or excise the web. ${ }^{3,4}$
However, the course of healing in our pediatric patient may be different compared to adults. If we perform outright division, there could be a higher risk of re-adhesion. Moreover, the presence of reflux (swollen arytenoids) may hasten the recurrence of the web or contribute to development of another laryngeal pathology like laryngomalacia. ${ }^{3}$

We are not aware of any definite treatment protocol that applies to our patient. As such, our current plan is serial monitoring to determine when he will be a good candidate for surgery. At the very least, we want to see a resolution of signs of reflux that may increase the risk of recurrence post surgery. We have yet to determine our final plan for surgical intervention and the optimal timing for it, and are open to receiving your comments. 